\title{
Surrogates for reef fish connectivity when designing marine protected area networks
}

\author{
Michael Bode ${ }^{1, *}$, Paul R. Armsworth ${ }^{2}$, Helen E. Fox ${ }^{3}$, Lance Bode $^{4,5}$ \\ ${ }^{1}$ ARC Centre of Excellence for Environmental Decisions, School of Botany, University of Melbourne, Victoria, 3010, Australia \\ ${ }^{2}$ Department of Ecology and Evolutionary Biology, University of Tennessee, Knoxville, Tennessee, USA \\ ${ }^{3}$ Conservation Science Program, World Wildlife Fund United States, 1250 24th Street, NW, Washington, DC, USA \\ ${ }^{4}$ Sohar University, Sohar, Sultanate of Oman \\ ${ }^{5}$ School of Engineering and Physical Sciences, James Cook University, Townsville, Queensland, Australia
}

\begin{abstract}
Reef fishes and other marine species occur in patchily distributed benthic populations that are interlinked by a larval stage where individuals disperse throughout the pelagic environment. This larval connectivity will play a critical role in determining whether marine protected area (MPA) networks can effectively promote the persistence of increasingly exploited reef fish populations. However, the amount, direction and variation of this connectivity are unknown for most species in most reef ecosystems of conservation concern. Furthermore, connectivity data are difficult to obtain and expensive to measure. Here, we demonstrate that if MPA locations are chosen according to certain easily measurable reef characteristics - 'connectivity surrogates' — the resulting MPA networks can maintain reef fish populations and allow fishery harvests superior to random expectation. Surrogates offer managers an opportunity to cheaply consider connectivity into MPA network design while data collection on connectivity is ongoing. We use a high-resolution biophysical model of reef fish larval connectivity on the Great Barrier Reef (GBR) to assess the effectiveness of 5 connectivity surrogates: 2 based on the reef's physical dimensions, 2 based on spawning biomass and 1 based on the efficient representation of conservation features. Biomass attributes generally perform best; however, the appropriate choice depends on the size of the proposed MPA network and the relative value placed on conservation outcomes and fisheries performance. Our results are relatively insensitive to the parameters used in the model and the morphology of the reef system. This robustness suggests that insights from the GBR could provide useful guidance to the management of other reef systems.
\end{abstract}

KEY WORDS: Coral trout $\cdot$ Plectropomus leopardus $\cdot$ Larval dispersal $\cdot$ Marine reserve planning

\section{INTRODUCTION}

Coral reefs are among the ocean's most sensitive ecosystems, with more than half already threatened by human activities (Halpern et al. 2008). A large proportion of coral reef fisheries - including irreplaceable protein sources for developing world communities - are currently undergoing dramatic declines or have already collapsed (Millennium Ecosystem Assessment 2005). Reef fisheries in developing nations face a large number of concurrent threats, including overharvesting, destructive fishing methods and coral bleaching (Halpern et al. 2008). Scientific consensus has concluded that marine protected areas (MPAs) should play a central role in achieving future global marine conservation goals (Lubchenco et al. 2003).

Almost all reef fish species exist in marine metapopulations (Roughgarden et al. 1985), with sessile adults and a dispersive pelagic larval stage that ranges from days to years (Leis 2002). Even within a single species, some larvae travel great distances during dispersal while others remain close to their natal reef (Jones et al. 2009). The potential for de- 
mographically significant inter-reef 'connectivity' means that populations can only be protected by networks of MPAs (Botsford et al. 2003, Cowen et al. 2006). Connectivity within MPA networks can support persistent populations in the face of threatening processes, while larval spillover from MPAs to fished reefs can increase populations and catches throughout a metapopulation. While the conservation importance of creating networks of MPAs has been acknowledged, incorporating connectivity into their design remains a difficult problem, since information on larval dispersal patterns is typically unavailable (Mora \& Sale 2002, Sale et al. 2005). Only in the last decade have useful connectivity data begun to appear from a range of sources. Mark-recapture techniques (Almany et al. 2007) and trace element analyses (Hamilton et al. 2006) can provide direct measurements of connectivity, while gene pool similarity and parentage (Jones et al. 2005) can help reconstruct recent larval dispersal events. Alternatively, hydrodynamic simulations of the advective current structure, coupled with larval behaviour models, allow the dispersal process to be simulated. These larval dispersal models have been created for a number of reef systems including the Caribbean (Cowen et al. 2006) and the Great Barrier Reef (GBR) (James et al. 2002). Of these methods, only larval dispersal modelling can provide connectivity data across the spatial and temporal scales relevant to conservation. Empirical techniques are resource and labor intensive, even when applied to a single year and reef. Coral reef ecosystems routinely contain hundreds or thousands of spawning and settlement sites, and connectivity is driven by processes that vary with periods as long as decades (e.g. ocean currents and atmospheric conditions; Robertson \& Allen 1996).

As well as being difficult to obtain, larval connectivity data complicate the MPA planning process. Demographic relationships between planning units (e.g. larval connectivity) add computational demands to already complex planning algorithms. Existing conservation prioritization methods therefore only consider connectivity in an ad hoc manner, through the use of adjacency scores or boundary length modifiers (Moilanen et al. 2009). These methods are unsatisfactory when MPAs are demographically dependent on ongoing connectivity, since they are not derived from, or explicitly related to, connectivity's role in shaping metapopulation dynamics. An alternative to these approaches is for conservation planning to use 'connectivity surrogates', reef-specific attributes that can help incorporate connectivity into the planning of MPA networks with limited, if any, knowledge about a reef system's connectivity. An effective surrogate would allow managers to plan MPA networks that protect connectivity simply by identifying reefs with the greatest amount of the surrogate. Because they are easier and cheaper to measure than connectivity itself, an effective surrogate could potentially allow connectivity to be costeffectively incorporated into management decisions, even as additional research into the precise form of the connectivity is ongoing. The purpose of this paper is to assess whether effective connectivity surrogates exist for reef fish on the GBR.

Candidate connectivity surrogates can be identified a priori, using an understanding of the processes that govern larval dispersal, or can be proposed on the basis of expert opinion. In either case, we assess the performance of proposed surrogates using 'virtual experiments' (Zurell et al. 2010). In a virtual experiment, we create a parameterised population viability analysis (PVA) model for our ecosystem of interest. We then apply each of our management alternatives to the PVA model and contrast the outcomes of repeated population simulations. Although the method does not clearly indicate why particular management actions perform better than others, it does provide a measure of their relative performance that has proven surprisingly robust to uncertainty (McCarthy et al. 2003). Virtual experiments have been used in fishery (Pauly et al. 2000, Sainsbury et al. 2000) and conservation management (MilnerGulland 1994, 2001), including, notably, by the International Whaling Commission (Cooke 1995). It is most effective when the dynamics of an ecosystem are well characterised (and a PVA model can therefore be constructed), but the scale of the system and the enormous number of potential control actions are too complex for the optimal management decision to be identified from first principles.

We use a new larval connectivity data set for the entire GBR to parameterise a virtual experiment for a species of coral trout Plectropomus leopardus, an economically valuable target reef fish species. Various connectivity surrogates have been proposed in the literature for reef fish (explicitly and implicitly), and we compare the performance of 5: 2 based on the physical dimensions of the reefs, 2 on their equilibrium biomass attributes and 1 on the representation of conservation features. For each surrogate, we create MPA networks with a range of sizes, under a range of plausible conditions, and assess the resulting networks from both a conservation and a fishery perspective. 


\section{MATERIALS AND METHODS}

For each of the surrogates, a subset of reefs is designated as an MPA network and protected from fishing pressure, while the remainder are fished. To assess surrogate performance, we simulate the PVA under a range of conditions and calculate the equilibrium population and the amount of fish harvested for each surrogate. We also perform the virtual experiment with MPAs chosen at random to ensure that the connectivity benefits we ascribe to the surrogates are not simply the result of protecting large proportions of reef habitat. Between each simulation, only the reefs included in the MPA network change, with total reef habitat protected, local dynamics and connectivity governed by the same equations. By assuming these factors to be constant, we allow the outcomes achieved by the different surrogates to be explained by their effect on metapopulation connectivity. One limitation of this approach, however, is that we are consequently unable to differentiate between 'population connectivity' (the number of successful settlers that disperse between 2 reefs) and 'reproductive population connectivity' (the number of dispersing individuals that survive to reproduce) (Pineda et al. 2007).

Our virtual experiment first requires an accurate reef fish population model for the GBR metapopulation. A model of any dispersing reef fish species must incorporate both the local population dynamics occurring on each reef and the metapopulation interactions that are mediated by larval connectivity. Second, the amount of each surrogate on every reef is calculated using the simulation model at the heart of the virtual experiment. For example, some of our surrogates protect reefs based on their biomass attributes. These attributes are themselves generated by the simulation model, and we are therefore able to define them endogenously. Third, we must define how reservation status affects fish population dynamics. Fourth, metrics must be identified that can gauge the performance of the different surrogates, from both conservation and fisheries perspectives. Finally, we must assess the robustness of the surrogate performance to various forms of uncertainty.

\section{Plectropomus leopardus metapopulation model}

Plectropomus leopardus is the main target of the GBR line fishing industry (Campbell et al. 2001) and is an important species for subsistence and export fisheries in many Southeast Asian nations (IUCN
2004). High fishing pressure has caused populations to decrease precipitously throughout its range, even in Australian fisheries, where controls are comparatively well enforced (Samoilys et al. 2001). Increasing fishing pressure and declining catch rates and populations have resulted in the IUCN classifying the species as 'near threatened' (Cornish \& Kiwi 2006).

At the reef scale, we model Plectropomus leopardus populations using a discrete time, age-structured model that only considers female individuals, assuming that males are reproductively saturating. We model the average density of coral trout on each reef, as dynamics are primarily regulated by densitydependent recruitment mortality resulting from limited available space. The density of female adult fish of a particular age $x$ on a particular reef $i$ is denoted $N_{i}^{x}(t)$ in year $t$. New individuals in the first age class are introduced to a reef as settling larvae; even offspring whose parents were members of the local population must undergo dispersal before selfrecruiting. Larvae that settle on each reef suffer density-dependent mortality according to a BevertonHolt relationship (Campbell et al. 2001). The density of settlers, $S_{i}$, that survive to enter the first age class of the adult population, $N_{i}^{1}$, is thus described by the function:

$$
N_{i}^{1}(t+1)=\frac{\alpha S_{i}(i)}{1+\beta S_{i}(t)}
$$

where $\alpha$ and $\beta$ are species-specific Beverton-Holt constants. The dynamics of the settlers that successfully recruit to the adult population are thereafter governed by density-independent survivorship, $S_{X}$ (Campbell et al. 2001), applied annually, so that:

$$
N_{i}^{X+1}(t+1)=s_{X} N_{i}^{X}(t)
$$

This mortality continues until the fish reach the age of senescence, $\omega$, at which point all remaining individuals die (i.e. $s_{\omega}=0$ ).

The individual populations are connected by larval dispersal. At each daylight high tide between October and December, fertilized eggs are released by reproductively mature females along the reef crest. These eggs are drawn into the pelagic environment by the ebbing tide, beginning a $7 \mathrm{~d}$ precompetent period after which locomotive capabilities develop. Once competent, the larvae remain active in the plankton for $3 \mathrm{wk}$, during which time they settle on the first reef habitat that enters their 'sensory zone', a region within $1 \mathrm{~km}$ of the competent larvae within which they can sense, and swim to, reefs. Dispersal is heavily influenced by currents and tides in and 
around the GBR lagoon, which vary interannually. The origins and destinations of larvae in a particular year $t$ are distilled into annual connectivity matrices $\boldsymbol{C}(t), 1996 \leq t \leq 2002$. Connectivity within a metapopulation of $M$ reefs is defined by a matrix $\boldsymbol{C}(t)$ which has $M^{2}$ elements. The matrix entry $c_{i j}(t)$ indicates the proportion of all the larvae spawned at reef $i$ that settle on reef $j$ in year $t$. For thorough methodological details of the biophysical model and connectivity matrices, see James et al. (2002).

The density of settlers in year $t$ on reef $j$ is defined by the equation:

$$
S_{j}(t)=\frac{1}{A_{j}} \sum_{i=1}^{M} c_{i j}(t) A_{i} \sum_{x=3}^{\omega} f_{x} N_{i}^{X}(t)
$$

where $A_{i}$ represents the amount of reef habitat on the $i$ th reef, and $f_{x}$ is the fecundity (i.e. the number of larvae released annually by each individual) of an $x$-yr-old adult female. The second summation therefore defines the larval output of all the reproductively mature females (age 3 or older) on each reef $i$, while the first summation calculates how many of these larvae arrive per-unit area on reef $j$. Eq. (3) couples the dynamics of the individual reefs (Eqs. 1 \& 2), creating a single interconnected metapopulation (assuming that the connectivity matrix for at least $1 \mathrm{yr}$ is irreducible; see Bode et al. 2006). The connectivity data set for the GBR comprises $7 \mathrm{yr}$; for each year, one connectivity matrix is chosen at random to simulate the effects of interannual variation. Metapopulation simulations are initialised with saturation densities of adults on each reef and are thereafter run for $500 \mathrm{yr}$, a sufficient length of time for the system to equilibrate.

Plectropomus leopardus is a comparatively wellstudied coral reef fish from a well-studied family (Pisces: Serranidae), allowing the metapopulation model to be informatively parameterised. The species' maximum recorded age is $\omega=14$, with individuals reaching sexual maturity at 3 yr of age (Ferreira \& Russ 1994). Density-independent post-settlement survivorship is low for juveniles $\left(s_{1}=0.6, s_{2}=0.7\right)$, but stabilises at a high value in the remaining years $\left(S_{\mathrm{x}}=\right.$ $0.83,3 \leq x \leq \omega)$ (Mapstone et al. 2004). The fecundity of mature female groupers can be estimated from their length, which in turn is a function of their age. The length of a female in $\mathrm{cm}$ in the $x$ th age class is (Ferreira \& Russ 1994):

$$
I_{X}=52.2\left(1-\mathrm{e}^{-0.354(x+0.766)}\right)
$$

with reproductive adults having fecundity (Sadovy 1996):

$$
f_{X}=13.82\left(l_{X}\right)^{3.03}
$$

The nonlinearity in these 2 relationships incorporates the disproportionate importance of older fish into the metapopulation dynamics (Birkeland \& Dayton 2005). The mass of an individual in kilograms can also be approximated using its length (Heemstra \& Randall 1993):

$$
z_{X}=0.0079\left(l_{X}\right)^{3.157}
$$

The 2 parameters of the Beverton-Holt post-settlement mortality model are not known for $P$. leopardus. We set $\alpha=1.5 \times 10^{-5}$ and $\beta=1 \times 10^{-8}$; these 2 values ensure that a high population is maintained across the system if every reef in the GBR is protected, but the metapopulation declines to extinction if all reefs are unprotected. This range of population sizes allows the performance of the surrogates to be differentiated. We test the robustness of the results to these 2 Beverton-Holt parameter assumptions below.

\section{Candidate connectivity surrogates}

The size of an MPA network is assessed by the proportion of reef habitat in the GBR that is protected. For each of the 5 surrogates, MPA networks are created by selecting reefs sequentially until the nominated proportion of reef habitat is protected.

\section{Surrogate 1: largest reefs}

James et al. (2002) showed that the removal of the reefs with the greatest area had a detrimental effect on metapopulation persistence, disproportionate to their total area. Hydrodynamic retention at the lee of large reefs may enhance self-recruitment (Black 1993), limiting spillover (Bartholomew et al. 2008). Area-controlled MPA networks consisting of the largest reefs will have comparatively few reserves, but increases in density, size and fecundity (Claudet et al. 2008) may compensate both conservation and fisheries for their small numbers. To construct an MPA network that protects a certain percent $(P \%)$ of total reef habitat using the largest reef surrogate, reef sizes are identified using satellite data, and the reefs are ranked from largest to smallest. We then repeatedly add the largest unprotected reef to the set of protected reefs until the required proportion of reef habitat is protected by MPAs. The result is that all protected reefs are larger than all unprotected reefs. 
Surrogate 2: smallest reefs

For a given size, an MPA network comprising the smallest reefs in the metapopulation contains the largest number of distinct MPAs. This surrogate will create an MPA network where, on average, reserves are close together and thus more likely to be able to exchange demographically significant amounts of larvae (Bohnsack 1998). Protecting the smallest reefs may have fishery benefits by increasing spillover and spreading its benefits more broadly across the metapopulation (Hastings \& Botsford 2003). However, the smallest reefs may have more difficulty securing settlers, especially through self-recruitment. MPA networks of small reefs are constructed in the same manner as the largest reef surrogate.

\section{Surrogate 3: reefs with the highest spawning biomass density}

In a given year, the production of larvae at a reef is determined by the spawning biomass of the local population. Reefs with a high density of biomass are therefore highly productive, and could potentially act as significant sources of larval spillover, increasing both populations and catches throughout the metapopulation. The biomass density on each reef could be measured with abundance surveys, or extrapolated from catch-effort data on reefs that are currently fished. To apply this surrogate in the virtual experiment, we measure the biomass density on each reef by simulating the GBR metapopulation without fishing until it reaches equilibrium.

\section{Surrogate 4: reefs with the highest} spawning biomass

Managers could also target the reefs with the highest total spawning biomass. This would obviously bias MPA sites towards the largest reefs, but this surrogate would also include smaller reefs that are disproportionately productive.

Surrogate 5: a habitat-representation-based MPA network

The current MPA network in the GBR is not based on connectivity but instead on design principles that focus primarily on efficiently representing the full range of habitat types, as well as the diversity of eco- logical communities and a small number of charismatic species (Fernandes et al. 2005). Nevertheless, it has been hypothesized that these methods may coincidentally protect connectivity within the network of MPAs (Leslie et al. 2003). We assess this hypothesis by calculating the performance of the current MPA network on the GBR, which protects $27 \%$ of reef habitat in the GBR. (This is slightly different from the $33 \%$ reported by the Great Barrier Reef Marine Park Authority, a value that also includes protected non-reef habitat.)

\section{Random reefs}

MPA networks, regardless of design, will increase the population of target species and therefore larval transport and spillover. For any of the connectivity surrogates listed above to be considered effective, they must enhance outcomes beyond the level we would expect simply from a given level of undirected protection. We therefore compare the performance of each of the connectivity surrogates with random expectation. For each of the following analyses, we compare each surrogate's performance with the range of outcomes generated by 100 different MPA networks of the same size, where protected reefs are selected at random. The distribution of random expectation is calculated for both conservation and fishery outcomes.

\section{Population dynamics on MPAs and fished reefs}

The effect that MPA designation has on reef fish population dynamics remains a subject of ongoing debate, but reviews have shown a consistent increase in populations of targeted species (Claudet et al. 2008, Lester et al. 2009). Plectropomus leopardus are pursued vigorously by commercial and recreational fisheries, and so MPAs will likely improve the density of exploited populations. Surveys on the GBR observed coral trout populations in MPAs that were several-fold greater than populations in non-MPAs, although this difference was regionally variable (Adams et al. 2000).

In our virtual experiment, populations in nonMPAs experience higher mortality $(m)$ than equivalent MPAs, which experience no additional mortality. The MPAs in our model are therefore no-take 'marine reserves', from which no resources can be removed. Each year a proportion $m_{i}$ of the adult fish populations on reef $i$ is removed from the system, in 
addition to the natural density-independent mortality. On protected reefs, $m_{i}=0$. Within the GBR Marine Park, a size restriction protects all individuals smaller than $38 \mathrm{~cm}$ (i.e. under $3 \mathrm{yr}$ ), and fishing mortality therefore only applies to individuals older than 2 yr. Campbell et al. (2001) estimate the fishing mortality on unprotected reefs in the GBR at $m_{i}=0.17$. With fishing affecting adults over the size limit, the metapopulation dynamics described by Eq. (3) become, for $x>2$ :

$$
N_{i}^{X+1}(t+1)=s_{X}\left(1-m_{i}\right) N_{i}^{X}(t)
$$

\section{Performance of the MPA networks}

The relative performance of the various connectivity surrogates is measured using 2 metrics. The first is the conservation outcomes generated by the MPA network, which we measure in a given year, $Q(t)$, by the total abundance of adult female Plectropomus leopardus across the GBR:

$$
Q(t)=\sum_{i=1}^{M} A_{i} \sum_{X=1}^{\omega} N_{i}^{X}(t)
$$

The second performance criterion is the outcome for fisheries. This is measured by $H(t)$, the total annual catch in tonnes:

$$
H(t)=\sum_{i=1}^{M} m_{i} A_{i} \sum_{x=3}^{\omega} z_{X} N_{i}^{X}(t)
$$

where the fishing intensity on reef $i, m_{i}$, is zero on reefs designated as MPAs. The second summation starts at $x=3$ to exclude the harvest of individuals below the length restriction. The stochasticity resulting from variable connectivity means that neither of these 2 measures is constant. We therefore show $50 \mathrm{yr}$ averages and allow the simulation to run for 450 yr before sampling to ensure that the choice of initial conditions does not affect our conclusions. Population abundances are therefore equilibrial, and the catches reported are sustainable.

\section{Testing the robustness of the connectivity surrogates}

Our primary results are derived from a base model that uses the best available estimates for the population parameters, applied to a metapopulation comprising all GBR reefs. However, if a particular surrogate is shown to effectively protect connectivity, 2 issues must be addressed. First, is surrogate perform- ance an artifact of our model assumptions, or of the specific characteristics of Plectropomus leopardus metapopulations? Second, are results particular to the GBR, or will they be applicable in other coral reef systems where modelled connectivity information is not available? We investigate these 2 questions by applying a set of 4 robustness analyses to the virtual experiment.

First, we assess the performance of the different connectivity surrogates over a range of different MPA network sizes, measured by the percentage of the total GBR reef habitat $P$ enclosed in MPAs. MPA networks are often designed to protect a predetermined percent of reef habitat in a region. For example, notake MPAs on the GBR made up $5 \%$ of the total marine park area before 2004 but now comprise $27 \%$ of the total reef habitat (Fernandes et al. 2005). We consider the performance of each surrogate across the range of possible MPA network sizes $(0-100 \%$ of GBR reef habitat, in intervals of $5 \%$ ). Second, we vary the values of $\alpha$ and $\beta$ in the Beverton-Holt relationship (Eq. 1), the only arbitrary life-history parameters in the model, by $\pm 50 \%$. Third, we consider whether the degree of protection offered by an MPA affects the best choice of surrogate. The amount of mortality $(m)$ experienced by unprotected populations is a parameter that can be influenced by off-reserve management actions, including regulations on gear, size and season length. We thus consider the impact of varying the value of $m$ between 0.085 and 0.17 (the estimated bounds provided by Campbell et al. 2001). In both these sensitivity analyses, the total MPA network size is held constant at $27 \%$.

Finally, it is important to assess whether surrogate performance is specific to the GBR case study. A surrogate whose effectiveness is limited to a single location cannot provide useful guidance to conservation in other regions. We can perform limited geographical sensitivity analyses by taking advantage of the GBR's varied regional morphology. Within 3 latitudinally defined subsections (each containing an equal amount of reef habitat), the GBR exhibits considerable variation along a number of character axes, including the width and depth of the continental shelf, the local reef density, the presence of contiguous barrier reef formations at the shelf break and the width of the GBR lagoon (Fig. 1). We repeat our analyses of the 5 candidate surrogates within each of these 3 subregions in isolation to assess whether this variation alters our conclusions.

Finally, we investigate whether the most effective connectivity surrogate is sensitive to the relative emphasis placed on fishery and conservation goals 


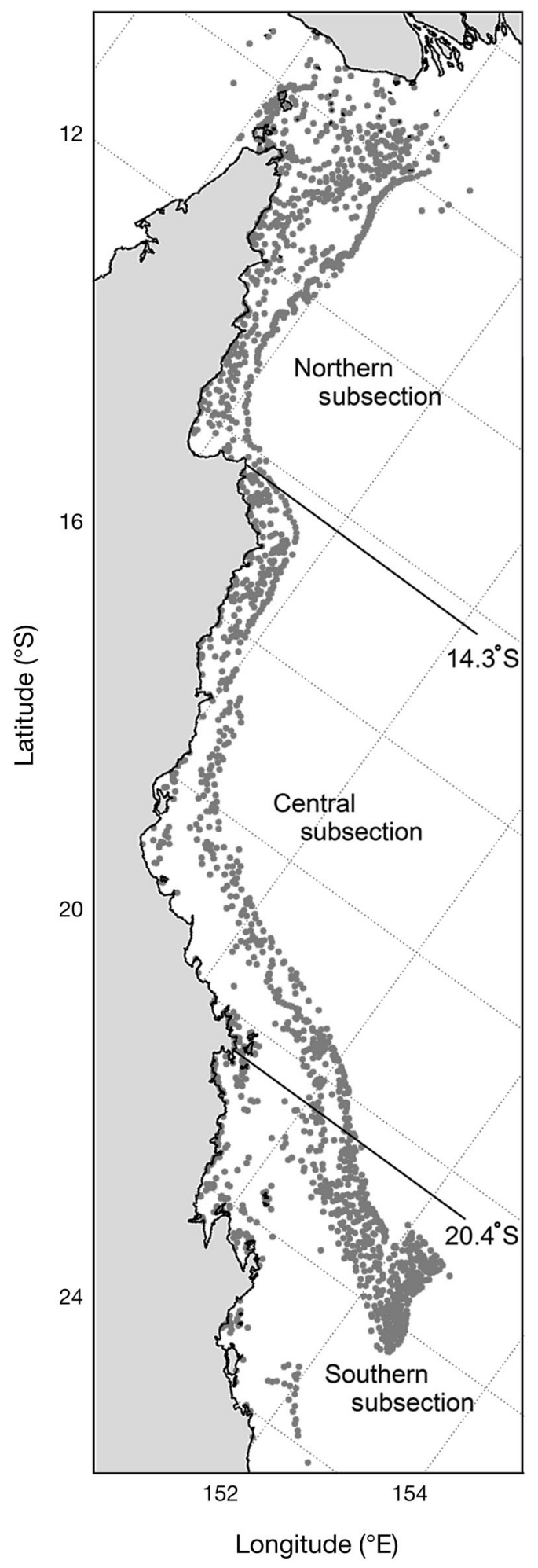

Fig. 1. The Great Barrier Reef metapopulation and location of the virtual experiments. Locations of the centroids of the 2175 reefs are marked by grey circles. Dark diagonal lines on the map demarcate 3 latitudinally defined subsections (northern, central and southern), each containing reefs with the same cumulative area by applying a straightforward multi-criteria decision analysis. Using a comparative weight $\kappa$, we construct an integrated performance metric, $B(\kappa, t)$, based on both fishery and conservation outcomes:

$B(\kappa, t)=\kappa \frac{\sum_{i=1}^{M} H_{i}(t)}{\check{H}}+(1-\kappa) \frac{\sum_{i=1}^{M} A_{i} \sum_{\check{x}=1}^{\omega} N_{i}^{\mathrm{x}}(t)}{\check{N}}$

where we use $\check{N}$ and $\check{H}$, the maximum total population abundance and maximum catch observed in the data set, respectively, to scale $N_{i}^{x}(t)$ and $H(t)$. We again consider time-averaged outcomes, calculating the mean value of $B(\kappa, t)$ over $50 \mathrm{yr}$. The parameter $\kappa$ reflects the weight that managers place onto fishery outcomes relative to conservation outcomes. If $\kappa=1$, managers consider only fishery outcomes, while $\kappa=0$ indicates that managers only value conservation. For values of $\kappa$ between 1 and 0 , we identify the connectivity surrogate that maximises the time-averaged value of $B$ for a range of potential MPA network sizes.

\section{RESULTS}

\section{Surrogate performance}

For the base analysis using the best-estimate parameter values, the conservation and fishery outcomes achieved by each of the surrogates are calculated across the entire GBR, and compared with random expectation (Fig. 2, Table 1). As the habitat representation surrogate is only defined for the existing GBR Marine Park, its performance is denoted with a single star at $P=0.27$ in Fig. 2 . We note that when our virtual experiment chose the existing MPA network on the GBR, the simulations using the best-estimate parameters generated a mean catch of $1970 \mathrm{t}$, which is of the same magnitude as the observed catches of the commercial coral trout fishery (approximately $1750 \mathrm{t}$ in 2003; Little et al. 2007).

Regardless of the connectivity surrogate applied, protecting ever-greater proportions of the reef system results in monotonically increasing conservation outcomes. Fishery outcomes, in contrast, are lowest when protection is complete $(P=100 \%)$ or absent $(P=$ $0 \%$, and peak at intermediate reserve network sizes. If too little reef habitat is protected, then equilibrium fish populations are too small to support a large catch. However, if the majority of reefs are protected, then the catch is also small, as few reefs are being made available for fishing. More interesting is the variation between connectivity surrogates in the location of the peak and the catch level that can be 


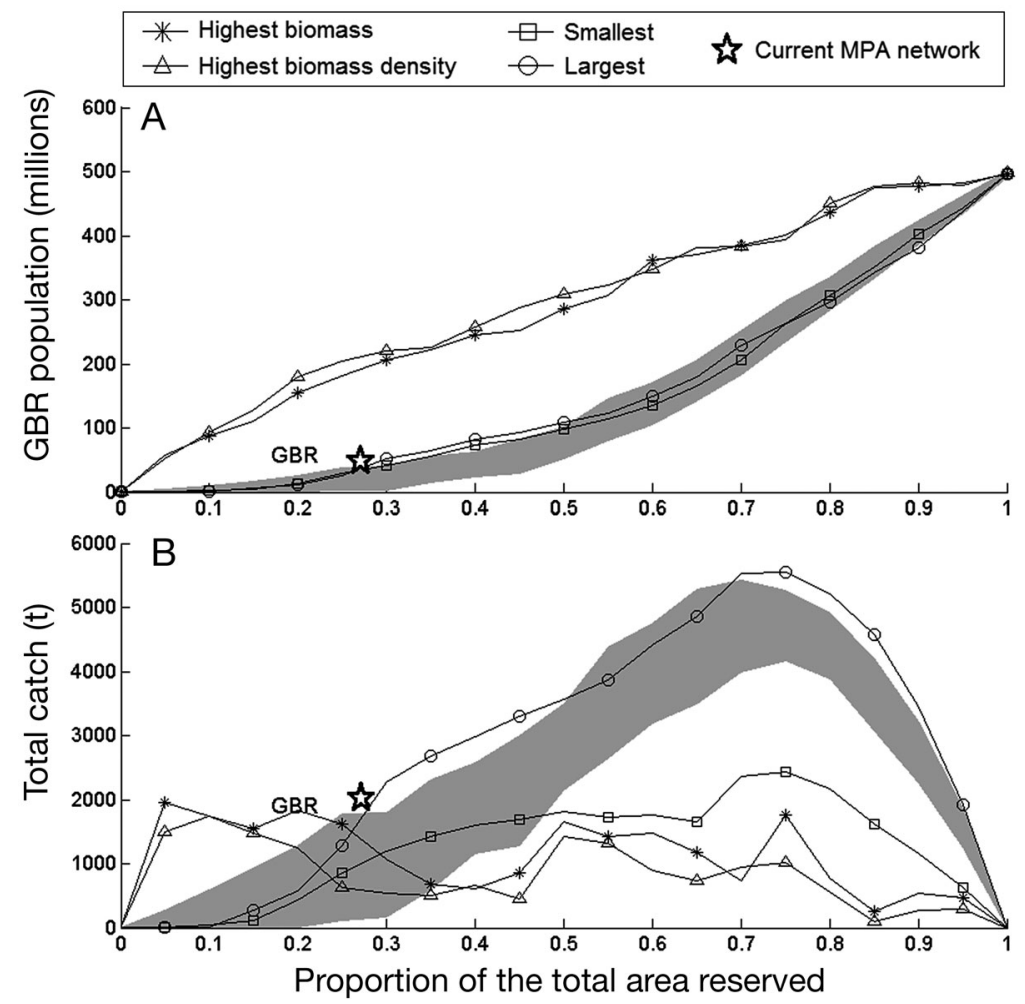

Fig. 2. Performance of the 5 connectivity surrogates for a range of total reserve sizes $(0 \leq P \leq 1)$, where $P$ measures the proportion of total reef area protected. (A) Total abundance of Plectropomus leopardus summed across all the reefs. (B) Total catch summed across all unprotected reefs. Results are at equilibrium. Grey bands indicate the range of outcomes resulting from a random choice of marine protected areas (MPAs). GBR:

Great Barrier Reef

supported. For conservation purposes, protecting either the biomass density of the candidate reefs or their total biomass produces outcomes that were much better than the remaining surrogates (Fig. 2A). Protecting reefs with the highest biomass density outperforms all alternative surrogates and random expectation for almost all MPA network sizes $P$. For conservation outcomes in MPA networks with $P<0.5$, both biomass-based surrogates generate populations that are 5 times larger than random expectation. These same surrogates also generate a greater equilibrium catch than random expectation in small MPA networks $0 \leq P \leq 0.2$ (Fig. 2B). However, both biomass-based surrogates provide substantially smaller catches than random expectation when $P \geq 0.35$.

Protecting the largest or the smallest reefs provides consistently poor conservation outcomes, supporting populations that are little different from random expectation regardless of the size of the MPA network (Fig 2A, Table 1). Nor do equilibrium catches encourage the use of size-based surrogates (Fig. 2B). Protecting the smallest reefs provides catches significantly lower than random expectation for all possible MPA network sizes. Protecting the largest reefs supports the greatest catches of any surrogate when $0.3 \leq P \leq$ 0.5 and when $P \geq 0.7$, but these outcomes fail to exceed random expectation by a substantial degree. Assessing the multicriteria performance of the surrogates (Eq. 10) across the range of possible MPA network sizes provides support for protecting reefs on the basis of their existing biomass (Fig. 3A). For typically sized MPA networks ( $\leq 30 \%)$, protection of the reefs with the highest biomass density generally ensures the best outcomes for the reef system,

Table 1. Inequalities indicate marine protected area (MPA) network sizes where connectivity surrogates (indicated by column headings) significantly outperform random expectation (i.e. lie outside the $95 \% \mathrm{CIs}$ ). $P$ : proportion of total reef area protected. Results are for both conservation $\left(P_{\mathrm{c}}\right)$ and fishery $\left(P_{\mathrm{e}}\right)$ objectives. GBR: Great Barrier Reef

\begin{tabular}{|c|c|c|c|c|}
\hline Reef system & Smallest & Largest & $\begin{array}{l}\text { Highest } \\
\text { biomass }\end{array}$ & $\begin{array}{l}\text { Highest biomass } \\
\text { density }\end{array}$ \\
\hline Full GBR & $\begin{aligned} & 0.35< P_{\mathrm{C}}<0.4 \\
&-\end{aligned}$ & $\begin{array}{c}0.3<P_{\mathrm{C}}<0.5 \\
0.3<P_{\mathrm{e}}<0.45 \text { and } \\
0.7<P_{\mathrm{e}}<0.95\end{array}$ & $\begin{array}{c}0<P_{\mathrm{c}}<1 \\
0<P_{\mathrm{e}}<0.2\end{array}$ & $\begin{array}{c}0<P_{\mathrm{c}}<1 \\
0<P_{\mathrm{e}}<0.2\end{array}$ \\
\hline $\begin{array}{l}\text { Northern } \\
\text { subsection }\end{array}$ & $\begin{array}{l}- \\
-\end{array}$ & $\begin{array}{l}0.15<P_{\mathrm{C}}<0.5 \\
0.15<P_{\mathrm{e}}<0.4\end{array}$ & $\begin{array}{c}0<P_{\mathrm{c}}<1 \\
0<P_{\mathrm{e}}<0.15\end{array}$ & $\begin{array}{c}0<P_{\mathrm{c}}<1 \\
0<P_{\mathrm{e}}<0.15\end{array}$ \\
\hline $\begin{array}{l}\text { Central } \\
\text { subsection }\end{array}$ & $\begin{array}{l}- \\
-\end{array}$ & $0.70<P_{\mathrm{e}}<0.85$ & $\begin{array}{c}0.1<P_{\mathrm{C}}<0.7 \text { and } 0.7<P_{\mathrm{c}} \leq 1 \\
0<P_{\mathrm{e}}<0.6\end{array}$ & $\begin{array}{c}0<P_{\mathrm{c}}<1 \\
0<P_{\mathrm{e}}<0.45\end{array}$ \\
\hline $\begin{array}{l}\text { Southern } \\
\text { subsection }\end{array}$ & - & $\begin{array}{c}P_{\mathrm{C}}=0.3 \\
0.9<P_{\mathrm{e}}<1\end{array}$ & $\begin{array}{c}0<P_{\mathrm{C}}<0.65 \text { and } \\
0.8<P_{\mathrm{C}}<1 \\
0.1<P_{\mathrm{e}}<0.35 \text { and } P_{\mathrm{e}}=0.4\end{array}$ & $\begin{array}{c}0<P_{\mathrm{C}}<0.7 \text { and } \\
0.8<P_{\mathrm{c}}<1 \\
0.1<P_{\mathrm{e}}<0.35 \text { and } P_{\mathrm{e}}=0.4\end{array}$ \\
\hline
\end{tabular}



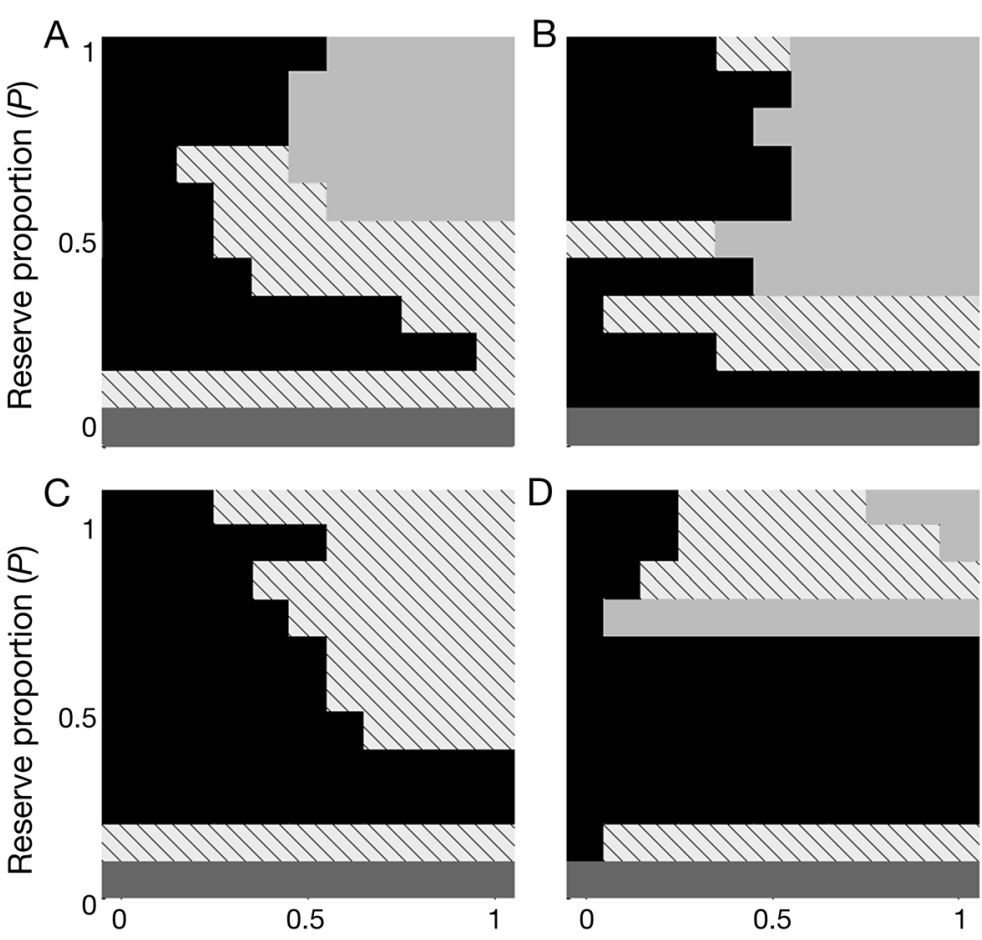

Relative objective weight ( $\kappa)$

Highest biomass

Highest biomass density

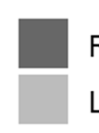

Random expectation

Largest reefs

Fig. 3. Identity of the best connectivity surrogate for a range of marine protected area (MPA) network sizes, $P$ (proportion of total reef area protected), and a range of relative weightings for conservation and fishery outcomes, $\kappa$. Results are shown for (A) the entire Great Barrier Reef metapopulation, (B) the northern subsection, (C) the central subsection and (D) the southern subsection

regardless of the relative weight placed on conservation versus fishery performance. If managers are conservation-focused $(\kappa<0.5)$, MPA networks should use the biomass or biomass density surrogate. For large MPA networks ( $\geq 50 \%$ ) that emphasise fishery harvests $(\kappa>0.5)$, protecting the largest reefs provides the best outcome of the surrogates considered here.

\section{Robustness analyses}

The relative performance of the different surrogates does not depend on our choice of parameters $\alpha$ and $\beta$, nor the intensity of fishing mortality $m$ on the unprotected reefs. Although varying the values of $\alpha$ and $\beta$ by $\pm 50 \%$, and $m$ between 8.5 and $17 \%$, alters the absolute fishery and conservation outcomes of the different surrogates, the 2 biomass-based surrogates sustain greater equilibrium populations than both random expectation and the size-based surrogates for all values of $P$. These 2 surrogates also allow the largest catches for low values of $P$, while the size-based surrogates still fail to substantially outperform random expectation.

The qualitative results also demonstrate some robustness to variation in the geography of the metapopulation. When the surrogate analysis is repeated for the 3 GBR subsections independently (Fig. 1), we find that the relative performance of the connectivity surrogates is qualitatively consistent (Fig. 3B-D). In all 3 subsections, the 2 biomass-based surrogates again perform much better than random expectation, and better than the 2 size-based surrogates, according to both fishery and conservation goals for MPA networks of $P \leq 30 \%$. The biomass surrogates provide consistently better than random catches for smaller MPA networks, but worse than random expectation for larger networks.

\section{DISCUSSION}

The results of these analyses cautiously support the potential of surrogates as tools for incorporating connectivity into MPA network design. Of the surrogates we examined, the existing biomass characteristics of the reefs provide the best indication of their role in transporting larvae around reef fish metapopulations in the absence of information on connectivity. For typically sized MPA networks $(P \leq 30 \%)$, targeting reefs with high biomass attributes generally results in better-than-expected performances from both a conservation and fishery perspective. This conclusion is insensitive to the model parameterisation, and robust to the regional morphology of the reef system. Approaching connectivity surrogates using a virtual experiment approach allowed the performance of different candidates to be measured under a range of different conditions, over realistic spatial scales. Although the connectivity patterns we used to model the coral trout metapopulation are of undetermined accuracy, they reflect the level of complexity and stochasticity in the GBR larval dispersal patterns better than any current alternatives.

The biomass-based surrogates are particularly effective when the total reserve size is small $(P<$ 
$15 \%$, a value similar to many existing MPA networks). In these situations, MPA networks that protect reefs with elevated biomass density support relatively high populations in conditions where the alternatives are completely ineffective. These MPAs are exporting larvae to the fished reefs, as evidenced by the greater-than-expected harvests achieved. A change in the network of MPAs could therefore boost targeted fish populations on both protected and unprotected reefs. When $P=20 \%$, for example, the surrogate protecting reefs on the GBR with the highest biomass performs much better than random expectation, supporting populations at $31 \%$ of their theoretical maximum (i.e. when the entire system is protected; Fig. 2A), while MPA networks constructed using non-biomass surrogates or random selection cannot maintain any population. At the same level of protection, the MPA network that is constructed according to this surrogate can provide sustainable catches that are close to one-third of the observed maximum and significantly greater than that arising from random expectation (Fig. 2B).

In contrast, the performance (both fishery and conservation) of size-based surrogates was seldom better than random expectation, and never substantially better. These surrogates provide particularly poor outcomes when a small proportion of the GBR's reef habitat is protected. If the proportion increases above $P=70 \%$, the largest-reef surrogate provides much higher catches compared with the other surrogates, but only slightly more than random expectation. In this situation, fishing effort becomes concentrated on a large number of small reefs (since protection of the largest reefs leaves the smallest reefs unprotected), rather than a few large reefs of an equivalent area. The resultant high catches indicate that this situation provides more larval spillover from MPAs to fished reefs. However, current objectives for marine conservation aim to protect $30 \%$ of marine habitats in MPA networks (WCPA 2007). Few MPA networks are likely to exceed $50 \%$ of the total reef habitat in the near future, and this result therefore has limited relevance.

The current MPA network on the GBR - comprising reefs chosen primarily to maximise habitat representation rather than connectivity - proved slightly more effective at protecting the abundance of a target species than a random set of reefs of similar size. The resulting modelled fishery harvest was also marginally larger than random expectation. These results may be specific to the planning methods and objectives used by the GBR Marine Park, as we did not repeat the approach across a broader range of
MPA network sizes. However, the observed conservation performance of this connectivity surrogate refutes the contention that representing a broad range of ecological attributes will necessarily result in enhanced protection for metapopulation connectivity for a species of management interest.

While these results indicate that surrogates could potentially be used to include connectivity in MPA networks, there are 2 key limitations that must be emphasised. First, while these surrogates are effective at protecting connectivity, the performance of an MPA network will depend on a range of other demographic processes that are not included in our model. To isolate and explore the role of connectivity per se, our metapopulation model assumes that habitat quality does not vary between reefs and that postsettlement mortality of larvae does not depend on their origin. Where these factors vary between reefs, the surrogates we identify will ensure a large amount of successful larval transport between protected reefs, but may not necessarily ensure that these settling larvae survive to reproductive age. This would affect surrogate performance from both a conservation and fisheries perspective.

Second, it is not clear how these results should be integrated into MPA networks with more complex and multifaceted objectives. Four examples illustrate this:

(1) Our simulations focus on a single species, yet most reef ecosystems are the focus of multiple extractive commercial, recreational and subsistence industries. Coral trout comprise $30 \%$ of the total catch landed by Queensland's commercial line fishing fleet (Ferreira \& Russ 1994), but the GBR contains other important harvest industries whose needs will also influence the location of MPAs; the abundances of these species may also affect coral trout populations. The utility of these surrogates for other species is not known, but cannot be guaranteed given that dispersal abilities vary substantially among species (Shanks 2009). Even for a single species, conservation managers need to consider many other ecological factors, such as known genetic structure or variation in habitat suitability.

(2) We have only included limited social and economic factors into these virtual experiments. In particular, fisheries are interested in the efficiency of harvesting as well as the total sustainable harvest. The travelling distances to fished reefs will affect profitability, as will the actions of the fishing fleets themselves. Industries are well-known for altering their behaviour and spatial effort distribution in response to the creation of MPAs, with fishing effort 
often displaced from MPAs onto unprotected reefs (Halpern et al. 2004).

(3) Our performance metrics were aggregated across the entire ecosystem, whereas managers may also aim to ensure equitable access across different regions or fishing groups (e.g. recreational, traditional, and commercial).

(4) We implicitly assumed that data on each of the connectivity surrogates could be gathered at the same cost. In practice, different surrogates are informed by different data sets, and a superior surrogate would be both effective and based on data that could be gathered quickly and cheaply. The 2 surrogates based on the physical dimensions of the reefs will be much less expensive than the biomass-based surrogates (indeed, the data on the GBR are already freely available). In contrast, the more effective biomass-based connectivity surrogates would be very expensive to define for each reef in a metapopulation.

Larval connectivity plays a key role in the population dynamics of many threatened and exploited marine organisms that could benefit from MPAs. The utility of this observation is limited, however, as connectivity data are unavailable for most species in almost all locations, and would be enormously expensive to gather. Effective connectivity surrogates would therefore deliver tremendous benefits to marine conservation decision-making by allowing managers to protect connectivity by targeting easily and cheaply measurable reef features. Virtual experiments using a number of candidate surrogates were performed on an economically important reef fish species, using a regional-scale metapopulation model parameterised by realistic larval connectivity patterns. The surrogates we applied were identified in previous research as being potentially important for connectivity, but many more candidates are available. The results of this study are complicated but encouraging. Despite varying extensively among even this limited data set, it was possible to obtain conservation and fishery outcomes that were better than random expectation for most potential MPA network sizes. By protecting reefs with the highest biomass attributes, MPA networks that protect 10 to $20 \%$ of the GBR's reef habitat could support elevated coral trout abundance and harvests. Importantly, the robustness of the surrogates' performance, both to parameter uncertainty and to the morphological characteristics of the reef system, offers hope that some information on connectivity can be incorporated into marine conservation planning in the absence of direct measurements. While the resulting management will not perform as well as decisions made with accurate knowledge of local connectivity patterns, they can provide immediate information even as the expensive and time-consuming process of connectivity data-gathering proceeds.

Acknowledgements. This work benefited from support from the National Institute for Mathematical and Biological Synthesis, an institute sponsored by the National Science Foundation (NSF), the US Department of Homeland Security, and the US Department of Agriculture through NSF Award no. EF-0832858. Support for M.B. was provided by the Australian Government's National Environmental Research Program, and the Australian Research Council Centre of Excellence for Environmental Decisions. The authors thank M. James and L. Mason for their work compiling the GBR connectivity data set.

\section{LITERATURE CITED}

Adams S, Mapstone BD, Russ GR, Davies CR (2000) Geographic variation in the sex ratio, sex specific size and age structure of Plectropomus leopardus (Serranidae) between reefs open and closed to fishing on the Great Barrier Reef. Can J Fish Aquat Sci 57:1448-1458

Almany GR, Berumen ML, Thorrold SR, Planes S, Jones GP (2007) Local replenishment of coral reef fish populations in a marine reserve. Science 316:742-744

Bartholomew A, Bohnsack JA, Smith SG, Ault JS, Harper DE, McClellan DB (2008) Influence of marine reserve size and boundary length on the initial response of exploited reef fishes in the Florida Keys National Marine Sanctuary, USA. Landscape Ecol 23:55-65

> Birkeland C, Dayton PK (2005) The importance in fishery management of leaving the big ones. Trends Ecol Evol 20:356-358

Black KP (1993) The relative importance of local retention and inter-reef dispersal of neutrally buoyant material on coral reefs. Coral Reefs 12:43-53

Bode M, Bode L, Armsworth PR (2006) Larval dispersal reveals regional sources and sinks in the Great Barrier Reef. Mar Ecol Prog Ser 308:17-25

Bohnsack JA (1998) Application of marine reserves to reef fisheries management. Aust J Ecol 23:298-304

Botsford LW, Micheli F, Hastings A (2003) Principles for the design of marine reserves. Ecol Appl 13:25-31

Campbell RA, Mapstone BD, Smith ADM (2001) Evaluating large-scale experimental designs for management of coral trout on the Great Barrier Reef. Ecol Appl 11: 1763-1777

> Claudet J, Osenberg CW, Benedetti-Cecchi L, Domenici P and others (2008) Marine reserves: size and age do matter. Ecol Lett 11:481-489

Cooke JG (1995) The International Whaling Commission's revised management procedure as an example of a new approach to fisheries management. In: Blix AS, Walloe L, Ulltang $O$ (eds) Whales, seals, fish and man. Elsevier, Amsterdam, p 647-658

Cornish A, Kiwi LK (2006) Plecropomus leopardus. Species report for the IUCN Red List of Threatened Species. IUCN, Gland

Cowen RK, Paris CB, Srinivasan A (2006) Scaling of connectivity in marine populations. Science 311:522-527 
Fernandes L, Day J, Lewis A, Slegers S and others (2005) Establishing representative no-take areas in the Great Barrier Reef: large-scale implementation of theory on marine protected areas. Conserv Biol 19:1733-1744

Ferreira BP, Russ GR (1994) Age validation and estimation of growth rate of the coral trout, Plectropomus leopardus, from Lizard Island, northern Great Barrier Reef. Fish Bull 92:46-57

> Halpern BS, Gaines SD, Warner RR (2004) Confounding effects of the export of production and the displacement of fishing effort from marine reserves. Ecol Appl 14: 1248-1256

Halpern BS, Walbridge S, Selkoe KA, Kappel CV and others (2008) A global map of human impact on marine ecosystems. Science 319:948-952

Hamilton SL, White JW, Caselle JE, Swearer SE, Warner RR (2006) Consistent long-term spatial gradients in replenishment for an island population of a coral reef fish. Mar Ecol Prog Ser 306:247-256

> Hastings A, Botsford LW (2003) Comparing designs of marine reserves for fisheries and for biodiversity. Ecol Appl 13:65-70

Heemstra PC, Randall JE (1993) FAO species catalogue, Vol 16. Groupers of the world (Family: Serranidae, Subfamily Epinephelinae). An annotated and illustrated catalogue of the grouper, rockcod, hind, coral grouper and lyretail species known to date. Food and Agriculture Organization of the UN, Rome

IUCN (2004) Leopard coral grouper. Species synopsis for the IUCN Red List. IUCN, Gland

> James MK, Armsworth PR, Mason LB, Bode L (2002) The structure of reef fish metapopulations: modelling larval dispersal and retention patterns. Proc R Soc Lond B Biol Sci 269:2079-2086

> Jones GP, Planes S, Thorrold SR (2005) Coral reef fish larvae settle close to home. Curr Biol 15:1314-1318

> Jones GP, Almany GR, Russ GR, Sale PF, Steneck RS, van Oppen MJH, Willis BL (2009) Larval retention and connectivity among populations of corals and reef shes: history, advances and challenges. Coral Reefs 28:307-325

- Leis JM (2002) Pacific coral-reef fishes: the implications of behaviour and ecology of larvae for biodiversity and conservation, and a reassessment of the open population paradigm. Environ Biol Fishes 65:199-208

Leslie H, Ruckelshaus M, Ball IR, Andleman S, Possingham HP (2003) Using siting algorithms in the design of marine reserve networks. Ecol Appl 13:185-198

Lester SE, Halpern BS, Grorud-Colvert K, Lubchenco J and others (2009) Biological effects within no-take marine reserves: a global synthesis. Mar Ecol Prog Ser 384: $33-46$

Little LR, Begg GA, Goldman B, Ellis N and others (2007) Modelling multi-species targeting of fishing effort in the Queensland coral reef fin fishery. Fishing and Fisheries Research Centre, Townsville

Lubchenco J, Palumbi SR, Gaines SD, Andelman S (2003)

Editorial responsibility: Romuald Lipcius,

Gloucester Point, Virginia, USA
Plugging a hole in the ocean: the emerging science of marine reserves. Ecol Appl 13:3-7

Mapstone BD, Davies CR, Little LR, Punt AE and others (2004) The effects of line fishing on the Great Barrier Reef and evaluations of alternative potential management strategies. Reef CRC, Townsville

McCarthy MA, Andelman SJ, Possingham HP (2003) Reliability of relative predictions in population viability analysis. Conserv Biol 17:982-989

Millennium Ecosystem Assessment (2005) Ecosystems and human well-being: synthesis. Island Press, Washington, $\mathrm{DC}$

Milner-Gulland EJ (1994) A population model for the management of Saiga antelope. J Appl Ecol 31:25-39

> Milner-Gulland EJ (2001) A dynamic game model for the decision to join an aggregation. Ecol Model 145:85-99

Moilanen A, Wilson KA, Possingham HP (eds) (2009) Spatial conservation prioritization. Oxford University Press, Oxford

> Mora C, Sale PF (2002) Are populations of coral reef fish open or closed? Trends Ecol Evol 17:422-428

- Pauly D, Christensen V, Walters CJ (2000) ECOPATH, ECOSIM and ECOSPACE as tools for evaluating ecosystem impacts on fisheries. ICES J Mar Sci 57:697-706

Pineda J, Hare JA, Sponaungle S (2007) Larval transport and dispersal in the coastal ocean and consequences for population connectivity. Oceanography 20:22-39

Robertson DR, Allen GR (1996) Zoogeography of the shorefish fauna of Clipperton Atoll. Coral Reefs 15:121-131

> Roughgarden J, Iwasa Y, Baxter C (1985) Demographic theory for an open marine population with space-limited recruitment. Ecology 66:54-67

Sadovy YJ (1996) Reproduction of reef fishery species. In: Polunin NVC, Roberts CM (eds) Reef fisheries. Chapman \& Hall, London, p 15-59

Sainsbury KJ, Punt AE, Smith ADM (2000) Design of operational management strategies for achieving fisheries ecosystem objectives. ICES J Mar Sci 57:731-741

Sale PF, Cowen RK, Danilowicz BS, Jones CG and others (2005) Critical science gaps impede use of no-take fishery reserves. Trends Ecol Evol 20:74-80

Samoilys MA, Squire L, Roelofs A (2001) Long-term monitoring of coral trout spawning aggregations on the Great Barrier Reef: implications for fisheries management. Abstract 2001 Indo-Pacific Fish Conference, May 20-25, Durban, South Africa. Oceanographic Research Institute, Durban

Shanks AL (2009) Pelagic larval duration and dispersal distance revisited. Biol Bull 216:373-385

WCPA (World Commission on Protected Areas) (2007) Establishing marine protected area networks: a guide for developing national and regional capacity for building MPA networks. IUCN, Gland

Zurell D, Berger U, Cabral JS, Jeltsch F and others (2010) The virtual ecologist approach: simulating data and observers. Oikos 119:622-635

Submitted: August 8, 2011; Accepted: July 3, 2012

Proofs received from author(s): October 8, 2012 